\title{
Corrigendum \\ Glutamate Receptors in Extinction and Extinction-Based Therapies for Psychiatric Illness
}

\section{Karyn M Myers, William A Carlezon Jr and Michael Davis}

Neuropsychopharmacology (201 I) 36, 910; doi:10.1038/npp.2011.3

Correction to: Neuropsychopharmacology Reviews (2011) 36, 274-293; doi:10.1038/npp.2010.88; published online 14 July 2010

In Table 2 of this article, the authors have corrected the data in the Effect column relating to Mao et al (2006), Mao et al
(2008), and Lin et al (2009) references. The revised table is shown below.

TABLE 2 Studies Employing NMDA Receptor Agonists or Other Positive Modulators

\begin{tabular}{|c|c|c|c|c|c|c|c|}
\hline Species & Test & Drug type & Drug, dose & Locus & Time of admin. & Effect & Reference \\
\hline \multirow[t]{3}{*}{ Rat } & FC-cue-FPS & Partial ag. & DCS, $3.25-30 \mathrm{mg} / \mathrm{kg}$ & Sys. & Pre-ext & Facilitated ext ret. & Walker et al. (2002) \\
\hline & & & DCS, $10 \mu \mathrm{g} / \mathrm{side}$ & BLA & Pre-ext & Same as systemic & \\
\hline & & Antag. + partial ag. & $\begin{array}{l}( \pm) H A-966,6 \mathrm{mg} / \mathrm{kg}+ \\
\text { DCS, } 15 \mathrm{mg} / \mathrm{kg}\end{array}$ & Sys. & Pre-ext & Co-admin of antag. blocked DCS effect & \\
\hline \multirow[t]{2}{*}{ Rat } & FC-cue-freezing & Partial ag. & $\mathrm{DCS}, 2.5-10 \mathrm{mg} / \mathrm{kg}$ & Sys. & Pre-or post-ext up to $2 \mathrm{hrs}$ & Facilitated ext ret. & Ledgerwood et al. (2003) \\
\hline & & & $\overline{\mathrm{DCS}, 10 \mu \mathrm{g} / \mathrm{side}}$ & BLA & Immed. post-ext & Same as systemic & \\
\hline Rat & FC-cue-freezing & Partial ag. & DCS, 15 mg/kg & Sys. & Immed. post-ext & Facilitated ext ret;; impaired reinst. & Ledgerwood et al. (2004) \\
\hline$\overline{\text { Rat }}$ & FC-cue-freezing & Partial ag. & DCS, $15 \mathrm{mg} / \mathrm{kg}$ & Sys. & Immed. post-ext & $\begin{array}{l}\text { Generalized ext: DCS + ext to Cue } 1 \text { facilitated ext ret. to Cue } 1 \\
\text { and Cue } 2\end{array}$ & Ledgerwood et al.( 2005) \\
\hline Rat & FC-cue-freezing & Partial ag. & $\mathrm{DCS}, 15 \mathrm{mg} / \mathrm{kg}$ & Sys. & Immed. post-ext & $\begin{array}{l}\text { Facilitated ext; tolerance to DCS after multiple admins; tolrance } \\
\text { dissipated over time }\end{array}$ & Parnas et al. (2005) \\
\hline Rat & FC-cue-FPS & Partial ag. & DCS, $15 \mathrm{mg} / \mathrm{kg}$ & Sys. & Pre-ext & $\begin{array}{l}\text { Facilitated ext ret.; effect blocked by MAPK or PI3K antagonist, } \\
\text { transciptional inhibitor, or protein synthesis inhibitor }\end{array}$ & Yang and Lu (2005) \\
\hline Rat & FC-cue-CER & Partial ag. & $\mathrm{DCS}, 15$ or $30 \mathrm{mg} / \mathrm{kg}$ & Sys. & Pre-ext & Facilitated ext; intact renewal & Woods and Bouton (2006) \\
\hline Rat & FC-cue-freezing & Partial ag. & DCS, $15 \mathrm{mg} / \mathrm{kg}$ & Sys. & Pre-ext & Facilitated ext ret. & Lee et al. (2006) \\
\hline Rat & FC-cue-FPS & Partial ag. & $\begin{array}{l}\text { DCS, } 10 \mu \mathrm{g} / \text { side } \\
\text { DCS, } 10 \mu \mathrm{g} / \mathrm{side}\end{array}$ & $\begin{array}{l}\text { BLA } \\
\text { BLA }\end{array}$ & $\frac{\text { Pre-ext }}{\text { Pre-ext }}$ & $\begin{array}{l}\text { Same as systemic } \\
\text { Facilitated ext ret. and reversed fear conditioning-induced increase } \\
\text { in BLA cell surface GluR1 expression. }\end{array}$ & Mao et al. (2006) \\
\hline Mouse & Inhib. avoidance & Partial ag. & DCS $15 \mathrm{mg} / \mathrm{kg}$ & Sys. $\times 15 \mathrm{~d}$ & Pre-ext & $\begin{array}{l}\text { Faciliated ext. in low or intermed. anxious mice but not in high } \\
\text { anxious }\end{array}$ & Tomilenko et al. (2007) \\
\hline Rat & FC-cue-freezing & Partial ag. & $\mathrm{DCS}, 15 \mathrm{mg} / \mathrm{kg}$ & Sys. & Immed. post-ext & $\begin{array}{l}\text { Facilitated ext. ret.; effect blocked by prior daily x } 14 \mathrm{~d} \text { admin of } \\
\text { DCS or imipramine }\end{array}$ & Werner-Seidler and Richardson (2007) \\
\hline Rat & FC-cue-freezing & Partial ag. & $\mathrm{DCS}, 15 \mathrm{mg} / \mathrm{kg}$ & Sys. & Immed. post-ext & Facilated ext ret. but only in rats showing some within-sess ext & Weber et al. (2007) \\
\hline \multirow[t]{2}{*}{ Rat } & FC-cue-FPS & Partial ag. & $\mathrm{DCS}, 15 \mathrm{mg} / \mathrm{kg}$ & Sys. & Pre-ext & Reversed disruption of ext ret. by glucocorticoid antag. & Yang and Lu (2007) \\
\hline & & & $\mathrm{DCS}, 5 \mathrm{mg} / \mathrm{kg}$ & Sys. & Pre-ext & Facilitated ext. ret: synergistic effect with low dose glucocorticoid & \\
\hline \multirow[t]{2}{*}{ Rat } & CTA & Partial ag. & & BLA & Pre-ext & Blocked impairment of ext ret. by muscimol, a GABA(A) receptor & Akirav (2007) \\
\hline & & & $\begin{array}{l}\text { Muscimol, } 0.05 \mu \mathrm{g} / \mathrm{side} \\
+ \text { + DCS, } 20 \mu \mathrm{g} / \mathrm{side}\end{array}$ & & & agonist & \\
\hline Rat & FC-cue-FPS & Partial ag. & DCS, $10 \mu \mathrm{g} /$ side & BLA & Pre-ext & $\begin{array}{l}\text { Facilitated ext ret. and reversed fear conditioning-induced increase } \\
\text { in BLA cell surface GluR1 expression; blocked reinst. }\end{array}$ & Mao et al. (2008) \\
\hline Rat & FC-cue-freezing, CER & Partial ag. & DCS 15 , or $30 \mathrm{mg} / \mathrm{kg}$ & Sys. & Pre-ext & $\begin{array}{l}\text { Facilitated ext ret. but only in rats showing some within-sess ext; } \\
\text { intact renewal }\end{array}$ & Bouton et al. (2008) \\
\hline Rat & FC-cue-FPS & Partial ag. & $\mathrm{DCS}, 15 \mathrm{mg} / \mathrm{kg}$ & Sys. & Pre-ext & $\begin{array}{l}\text { Facilitated 1st but not 2nd ext unless 2nd ext involved a different } \\
\text { cue }\end{array}$ & Langton and Richardson (2008) \\
\hline Mouse & FC-cue-freezing & Partial ag. & $\mathrm{DCS}, 5,15,30 \mathrm{mg} / \mathrm{kg}$ & Sys. & Pre-ext & Facilitated ext retention in C57BL/6J but not $129 \mathrm{~S} 1$ mice & Hefner et al. (2008) \\
\hline Rat & FC-cue-FPS & Partial ag. & DCS, $20 \mathrm{mg} / \mathrm{kg}$ & Sys. & Pre-ext & $\begin{array}{l}\text { Facilitated ext ret. and reversed fear conditioning-induced increase in BLA } \\
\text { AMPANNMDA receptor ratio; effects prevented by endocytosis blocker }\end{array}$ & Lin et al. (2009) \\
\hline Mouse & FC-cue-freezing & Partial ag. & $\mathrm{DCS}, 30 \mathrm{mg} / \mathrm{kg}$ & Sys. & Pre-ext & Facilitated ext ret.; impaired reinst.; no effect on 2nd ext & Yamada et al. (2009) \\
\hline Rat & CPA (morphine W/D) & Partial ag. & DCS, $15 \mathrm{mg} / \mathrm{kg}$ & Sys. & Pre-ext & Facilitated ext ret. & Myers and Carlezon (2010) \\
\hline$\overline{R a t}$ & FC-cue, ctx-freezing & $\begin{array}{l}\text { Pos. mod. via } \uparrow \mathrm{D} \text { - } \\
\text { serine }\end{array}$ & $\begin{array}{l}\text { Mutation in catabolic } \\
\text { enzyme for D-serine }\end{array}$ & Sys. & Constitutive & NE on FC; facilitated ext of freezing to context but not cue & Labrie et al. (2009) \\
\hline Rat & Inhib. avoidance & Partial ag. & Spermidine, $2 \mathrm{nmol}$ & Hipp. & Post-ext & $\begin{array}{l}\text { Drug admin. immed but not } 6 \text { hrs post-ext faciliated ext ret; effect } \\
\text { blocked by co-admin of NR2B antag. }\end{array}$ & Gomes et al. (in press) \\
\hline Rat & Bar press for food & Partial ag. & $\mathrm{DCS}, 3 \mathrm{mg} / \mathrm{kg}$ & Sys. & Pre-ext & Impaired within-sess ext.; no drug-free post-test; prob. perf. effect & Port and Seybold (1998) \\
\hline \multirow[t]{2}{*}{ Rat } & Cocaine cond. PP & Partial ag. & $\mathrm{DCS}, 15 \mathrm{mg} / \mathrm{kg}$ & Sys. $\times 9 \mathrm{~d}$ & Post-ext & $\begin{array}{l}\text { Facilitated ext if given immed. but not } 4 \text { hrs post-ext; blocked } \\
\text { relapse at } 2 \text { wks (longest interval tested) }\end{array}$ & Botreau et al. (2006) \\
\hline & & & DCS, $10 \mu \mathrm{g} / \mathrm{side}$ & BLA $\times 3 d$ & Post-ext & Same as systemic & \\
\hline Mouse & Cocaine cond. PP & Partial ag. & DCS, $15 \mathrm{mg} / \mathrm{kg}$ & Sys. & Pre-ext & $\begin{array}{l}\text { May have facilitated ext. and blocked spontaneous recovery; NE on } \\
\text { cocaine reinst. }\end{array}$ & Kelley et al. (2007) \\
\hline Rat & Amphet. PP & Partial ag. & DCS, $10 \mu \mathrm{g} / \mathrm{side}$ & d. hipp. & Pre-ext & Facilitated ext ret. but also facilitated reacq. & Sakurai et al. (2007) \\
\hline Rat & Run maze for food & Partial ag. & DCS, $15 \mathrm{mg} / \mathrm{kg}$ & Sys. & Immed. post-ext & Faciliated ext & Gabriele and Packard (2007) \\
\hline Rat & Ethanol IVSA & Partial ag. & DCS, $5 \mathrm{mg} / \mathrm{kg}$ & Sys. $\times 12 \mathrm{~d}$ & Pre-ext & Facilitated ext retention 1 day after last ext. session drug free & Vengeliene et al. (2008) \\
\hline Mouse & Bar press for food & Partial ag. & DCS, $15,30 \mathrm{mg} / \mathrm{kg}$ & Sys. $X 8 d$ or $10 d$ & Immed. post-ext & $\begin{array}{l}\text { Faciliated ext.; efficacy of DCS dependent on interval between ext } \\
\text { sessions }\end{array}$ & Shaw et al. (2009) \\
\hline Rat & Cocaine cond. PP & Partial ag. & $\mathrm{DCS}, 15 \mathrm{mg} / \mathrm{kg}$ & Sys. & Immed. post-ext & Facilitated ext across days; blocked relapse & Paolone et al. (2009) \\
\hline$\overline{R a t}$ & Cocaine IVSA & Agonist & D-serine, $100 \mathrm{mg} / \mathrm{kg}$ & Sys. & Pre- or post-ext & NE on ext rate; attenuated cocaine- but not sucrose-induced reinst. & Kelamangalath et al. (2009) \\
\hline Mouse & Ethanol PP & Partial ag. & $\mathrm{DCS}, 15-60 \mathrm{mg} / \mathrm{kg}$ & Sys. $\times 12 d$ & Pre-ext & NE on ext rate; impaired reacq & Groblewski et al. (2009) \\
\hline Mouse & Cocaine cond. PP & Partial ag. & DCS, $15,30 \mathrm{mg} / \mathrm{kg}$ & Sys. $\times 8 d$ & Immed. post-ext & $\begin{array}{l}\text { Facilitated ext; } 30 \mathrm{mg} / \mathrm{kg} \text { DCS relapse at } 2 \text { weeks compared to } \\
\text { saline or } 15 \mathrm{mg} / \mathrm{kg} \mathrm{DCS}\end{array}$ & Thanos et al. (2009) \\
\hline Rat & Cocaine IVSA & Partial ag. & $\mathrm{DCS}, 30 \mathrm{mg} / \mathrm{kg}$ & Sys. & Pre or post-ext & Facilitated ext when given immed but not 6 hrs post-ext; retarded reaca & Nic Dhonnchadha et al. (2009) \\
\hline Monkey & Cocaine IVSA & Partial ag. & $\mathrm{DCS}, 10 \mathrm{mg} / \mathrm{kg}$ & Sys. & Pre-ext & NE on ext; retarded reacq. & \\
\hline
\end{tabular}

Abbreviations: admin, administration; ag, agonist; amphet, amphetamine; antag, antagonist; BLA, basolateral amygdala; cond, conditioned; CPA, conditioned place aversion; CTA, conditioned taste aversion; ctx, context; d, dorsal; dep, dependent; ext, extinction; FC, fear conditioning: FPS, fear-potentiated startle; hipp, hippocampus; immed, immediate; inhib, inhibiton; IVSA, intravenous self-administration; mod, modulation, mPFC, medial prefrontal cortex; NE, no effeet; perf, performance; poss, possible; prob, probable; PP, place preference; pos, positive; reacq, reacquisition; reinst, reinstatement; ret, retention; sess, session; sys, systemic; vmPFC, ventromedial prefrontal cortex; W/D, withdrawal. 